\title{
DESIGNERS AND COLLABORATIVE VIRTUAL ENVIRONMENTS ${ }^{1}$
}

\author{
M L MAHER \\ University of Sydney, Australia
}

\begin{abstract}
This chapter provides an overview of the technical and social issues of CVEs and their impact on designers. The development of CVEs has lead to new ways for designers to collaborate and new kinds of places for designers to design. As a new technology for collaborative design, CVEs impact the collaborative process by facilitating movement between working together and working individually. As new technologies for interacting with CVEs include tangible interfaces, we can expect to see changes in the perception of the design that lead to changes in spatial focus.
\end{abstract}

Keywords. 3D Virtual Worlds. Collaborative design, tangible interaction, protocol studies, adaptive agents

\section{Introduction}

Collaborative Virtual Environments (CVEs) are virtual worlds shared by participants across a computer network. There are many descriptions of CVEs, and Benford et al (2001) provides one that is assumed in this chapter: The virtual world is typically presented as a 3D place-like environment in which participants are provided with graphical embodiments called avatars that convey their identity, presence, location, and activities to others. CVEs vary in their representational richness from $3 \mathrm{D}$ virtual reality to $2 \mathrm{D}$ images to text-based environments. The participants are able to use their avatars to interact with and sometimes create the contents of the world, and to communicate with one another using different media including audio, video, gestures, and text. This kind of virtual environment provides opportunities for collaborative design that

${ }^{1}$ Maher, M.L. (2010). Designers and Collaborative Virtual Environments, In Xinagyu Wang and Jerry Tsai (Eds) Collaborative Design in Virtual Environments, Springer. 
gives remote participants a sense of a shared place and presence while they collaborate.

CVEs provide new ways to meet communication needs when negotiation is important and frequent, and complex topics are being discussed. They provide more effective alternatives to video conferencing and teleconferencing because they provide spaces that explicitly include both data representations and users (Churchill et. al. 2001), an important consideration in collaborative design where the focus of a meeting may be on the design ideas and models more than on the faces of the collaborating designers. During the early days of CVEs (in the early 1990s), researchers put an emphasis on simulating face-to-face co-presence as realistically as possible (Redfern and Naughton, 2002). More recently, it has been realised that this is not enough for collaborative design, and may not necessarily even be required to develop a shared understanding of the design problem and potential solutions (Saad and Maher, 1996).

Redfern and Naughton (2002) nicely summarize a range of technical and social issues provided in the development and use of CVEs in distance education that can be adapted for understanding the development and use of CVEs in collaborative design.

Managing collaborative design processes. In a design project, collaborative work involves the interleaving of individual and group activities. Managing this process over the several months of a design project requires considerable explicit and implicit communication between collaborators. Individuals need to negotiate shared understandings of design goals, of design decomposition and resource allocation, and of progress on specific tasks. It is important that collaborators know what is currently being done and what has been done in context of the goals. In a collaborative design task this information can be communicated in the objects within the CVE where the collection of objects forms an information model, such as the Building Information Model in building design processes. DesignWorld is an example of a research project that explores ways of integrating CVEs with an external database of objects and project information (Maher et al 2006).

"What You See Is What I See" (WYSIWIS). Conversational and action analysis studies of traditional collaborative work have shown the importance of being able to understand the viewpoints, focuses of attention, and actions of collaborators. CVEs assume a co-presence in a 
Designers and Collaborative Virtual Environments

virtual world that is shared, even though the viewpoint of the world may be different when the avatars are located in different places and facing different directions. Communication among the participants in a CVE is often about location and viewpoints, allowing individuals to pursue their own tasks as well as have their attention focussed on a shared task. Clark and Maher (2006) studied communication in a design studio course that was held in a CVE and showed that a significant percentage of the communication was about location and presence.

Chance meetings. Informal meetings with colleagues are rarely provided for in collaborative tools, yet they are an important characteristic of the effectiveness of many workers, particularly knowledge-workers. Recent research has investigated mechanisms for supporting chance meetings without the requirement for explicit action by the user (McGrath $\&$ Prinz, 2001). In collaborative design, studies have shown that designers move fluidly from working individually to working together. Kvan (2000) presents a model in which different stages of collaborative design are characterized as closely coupled or loosely coupled. CVEs provide the opportunity for individual work in a shared place that supports chance meetings.

Peripheral awareness is increasingly seen as an important concept in collaborative work, as evidenced in ethnographic studies. Team members involved in parallel but independent ongoing activities need to be able to co-ordinate and inform their activities through background or peripheral awareness of one another's activities. The affordance of peripheral awareness for collaborative design in a CVE is demonstrated in a study done by Gul and Maher (2009). In this study, designers were given similar design tasks in a 3D CVE and in a remote sketching environment, and asked to collaborate for a fixed period of time. An analysis of the protocol data shows that in a 3D CVE designers were inclined to spend part of the time working together and part coordinating their individual work, while in a remote sketching environment the designers did not work individually.

Non-verbal communications are known to have a strong effect on how utterances are interpreted. Research into alternative input mechanisms for capturing this type of information from the user has been underway for some time: recently, attempts are being made to make these mechanisms intuitive and non-intrusive. Clark and Maher (2006) show how participants communicated using specified gestures for their avatars in the design 
studio. Augmented reality approaches to CVEs promise a more spontaneous integration of movement in the physical world being communicated in a virtual world.

The "designing for two worlds" principle: People are never fully immersed in a virtual world, but are always partially in the real world too. Certain activities when carried out in the real world have a very strong impact on the participant's activities that should be recognised in the virtual world - for example, answering the phone. DesignWorld (Maher et al 2006) accommodated this by maintaining a video of each designer in his physical office in a window adjacent to the 3D CVE with the designers' avatars. This allows communication to be directed in the virtual world or in the physical world, and the physical presence and activities of the physical world to be communicated to the designers in the virtual world.

This chapter provides an overview of two comparative studies of collaborating designers using CVE technologies. These studies provide a starting point for understanding the impact of these technologies on design cognition and design collaboration. The chapter ends with an overview of a project that considers the opportunities that CVEs provide for designers to explore a new kind of design discipline: the design of places in virtual worlds. These three projects consider designers more comprehensively in the context of CVEs: from designers as users of CVEs to designers of CVEs.

\section{Supporting Collaborative Design: From Sketching to CVEs}

Sharing design ideas ranges from working together at a table while sketching with paper and pencil, to working in a CVE. CVEs do not replace sketching on paper while co-located; they provide a different kind of environment for collaborating. Since the tools for expressing and sharing ideas are so different, we would expect that the collaboration is different. Gul and Maher (2009) describe a study comparing design collaboration while designers sit together sketching to remote sketching and designing in a 3D CVE. The aim of the study is to identify the changes in collaborating designers' behaviour and processes when they move from co-located sketching to remote designing.

The study considered three collaborative design settings: sketching around a table, remote sketching, and designing in a CVE. The sketching setting is shown in Table 1. The image in the left part of the table shows a 
camera image of two designers sharing a physical table with sketching tools such as paper and pencil. The schematic in the right part of the table shows how the designers were observed by placing 2 cameras connected to a digital video recorder.

TABLE 1. Sketching experimental setting (Gul and Maher, 2009)
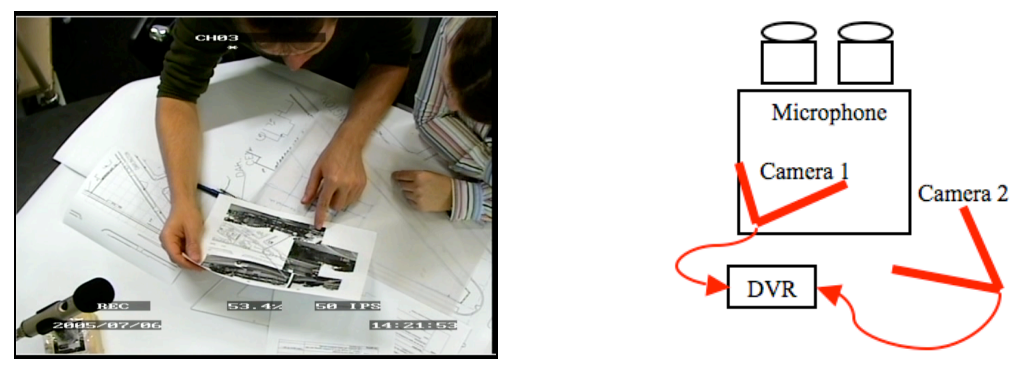

The remote sketching setting used GroupBoard (www.groupboard.com), a collaborative sketching application, and display surfaces with pen interfaces. One designer was sketching on a tabletop system and the other designer was sketching on a whiteboard mounted on the wall. The setting is shown in Table 2. The top row of the table shows the tabletop sketching environment (left) and the mounted whiteboard sketching environment (right). The bottom row of the table shows a schematic of the layout: the location of cameras for recording the design session, and the use of a panel to simulate remotely located designers.

Table 3 shows the applications for the two remote design sessions: the GroupBoard application supported a shared sketching window, as shown in Table 3(a) and the Active Worlds (www.activeworlds.com) application is a CVE, as shown in Table 3(b). The third setting was set up similarly to the second setting with cameras on each of the remotely located designers, as well as capturing the interactions on the display screen.

A protocol analysis method was used to compare face to face sketching to remote sketching and a CVE. The protocol data included video of the shared representation and gestures of the designers, and the verbal utterances of the designers. The continuous stream of protocol data was first segmented using the utterance-based segmentation method as used in (Gabriel 2000; Maher, Bilda and Marchant, 2005) where a new segment 
was marked when there was a shift from one designer acting/talking to another. When a segment contained complex actions, each segment was segmented again using the actions-and-intentions segmentation method used in (Gero and McNeill, 1998). The segments were coded using a hierarchical coding scheme according to perception, action, and realization categories, as well as codes for collaboration mode (meeting, individual) and representation mode (2D, 3D).

TABLE 2: Remote sketching setting (Gul and Maher, 2009)

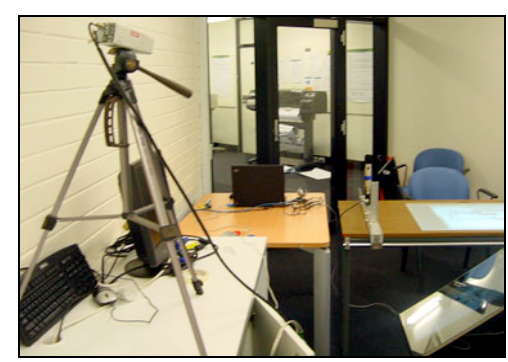

(a)

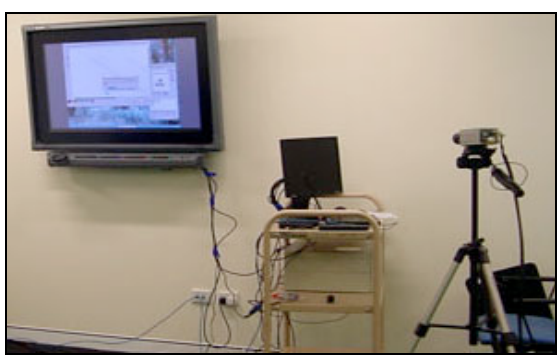

(b)

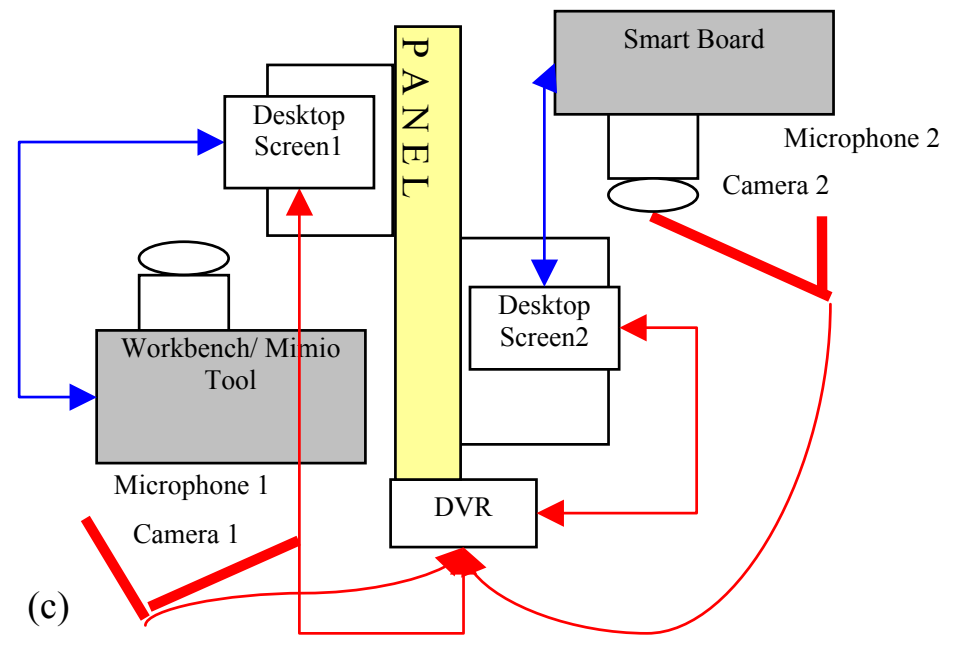


Designers and Collaborative Virtual Environments 
TABLE 3: Remote sketching and CVE interfaces (Gul and Maher, 2009)

(a) GroupBoard Interface

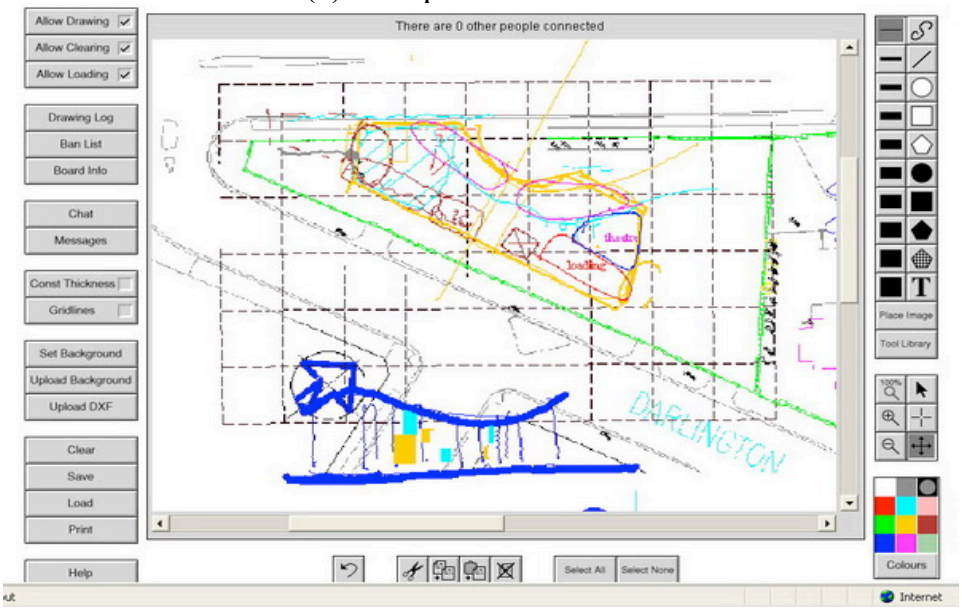

(b) ActiveWorlds Interface

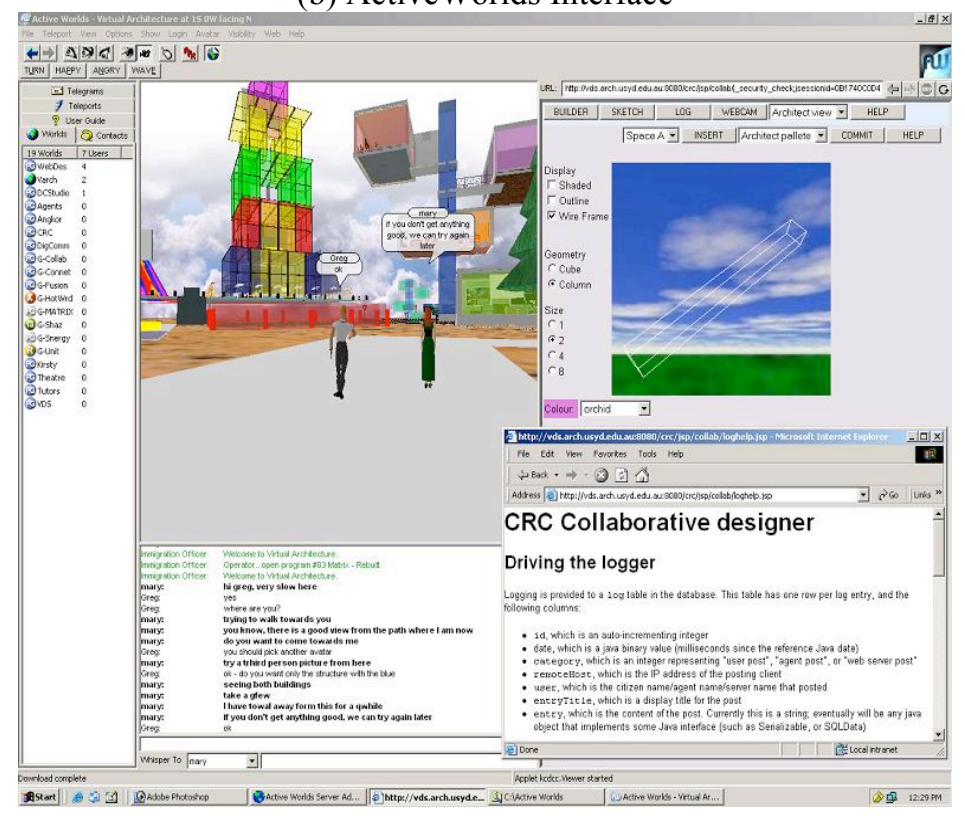


Designers and Collaborative Virtual Environments

An immediately notable result from the segmentation process was that the designers had more attention shifts when sketching face to face, that is, the average duration of the segments was shorter and there were more segments in a design session. In a CVE, the designers stayed focused on one activity longer and had fewer segments to complete the same design goals. When comparing the protocols for sketching vs working in $3 \mathrm{D}$, the study found that when sketching the designers did more "create" and "write" actions and when 3D modelling the designers did more "continue" actions which provided more detail in the co-created representation. The effect of facilitating more detailed digital representations is that the result of the remote 3D design sessions was a more developed design solution.

The second most notable result of this study was that the designers worked together continuously when sketching. They stayed focused on a common task. The designers in the CVE worked in two modes: together on the same task, and separately on individual tasks. The CVE provided awareness of the collaborator but also allowed the collaborating designers to have their own view of the design model and work independently, while checking in with each other occasionally.

\section{Adding Tangible Interaction to 3D Design}

The interaction with most CVEs is still enabled by the keyboard and mouse. Interaction technology is moving towards alternatives to the keyboard and mouse, such as pen interfaces, the Wii, direct braincomputer interaction, and tangible interaction. A study by Kim and Maher (2008) looks at the difference in the design process when designers use a mouse and keyboard vs 3D blocks as tangible input devices.

Tangible user interfaces (TUIs) offer physical objects as an alternative to typical computer input and output devices, and are often combined with augmented reality (AR) blending reality with virtuality (Azuma et al. 2001; Azuma 1997; Dias et al. 2002). Using a tabletop system, designers can interact with 3D digital models more directly and naturally using TUIs while still utilizing verbal and non-verbal communication (Bekker 1995; Scott et al. 2003; Tang 1991). Many researchers have proposed that tangible interaction combined with AR display techniques might affect the way in which designers perceive and interact with digital models (Billinghurst and Kato 2002; Streitz et al. 1999; Ullmer and Ishii 1997). However, the central preoccupation of research on TUIs has been in 
developing new prototypes for design applications focusing on the functionality, where the Kim and Maher (2008) study provides empirical evidence for the effect of TUIs on designers' spatial cognition.

This empirical study considers the effects of TUIs on designers' spatial cognition using a protocol analysis. In order to focus on the impact of tangible interfaces on designers' spatial cognition, two settings are compared: a graphical user interface (GUI) as a desktop computer with a mouse and keyboard in ArchiCAD, and a TUI as tabletop system with 3D blocks in ARToolkit.

The tabletop system was developed at the University of Sydney and is described in (Daruwala, 2004). The tabletop system is shown in Figure 1 The horizontal display provides the means on which tangible interaction can take place and the binary patterns of the 3D blocks were made in ARToolKit2 for the display of the 3D virtual models. A web camera captures the patterns and outputs them on a vertical LCD display in real time. In order to provide a same visual modality as the GUI environment, an LCD screen is used for the TUI session instead of a head mounted display (HMD).

Table 4 shows the set-up for the TUI session. The LCD screen was fixed to the left of the designers, and a 2D studio plan and 3D blocks were placed on the horizontal table. The web camera was set at a suitable height and angle to detect all the markers of the 3D blocks. A DVR (digital video recording) system was set to record two different views on one monitor, where one camera was used to monitor designers' behaviour and the other to capture the images on the LCD screen. This enabled the experimenter to observe designers' physical actions and the corresponding changes in the representation. A microphone was fed into the DVR system through a sound mixer and the camera filmed to record a clear view of designers' behaviours. A pair of designers sat at the same side of the table.

${ }^{2}$ ARToolKit is free AR software using a computer vision method and includes tracking libraries and source codes for the libraries, which is easy to use and allowed us to customise existing codes for our own applications (Billinghurst et al. 2003). 
TABLE 4. Experimental set-up for the TUI session (Kim and Maher, 2008)
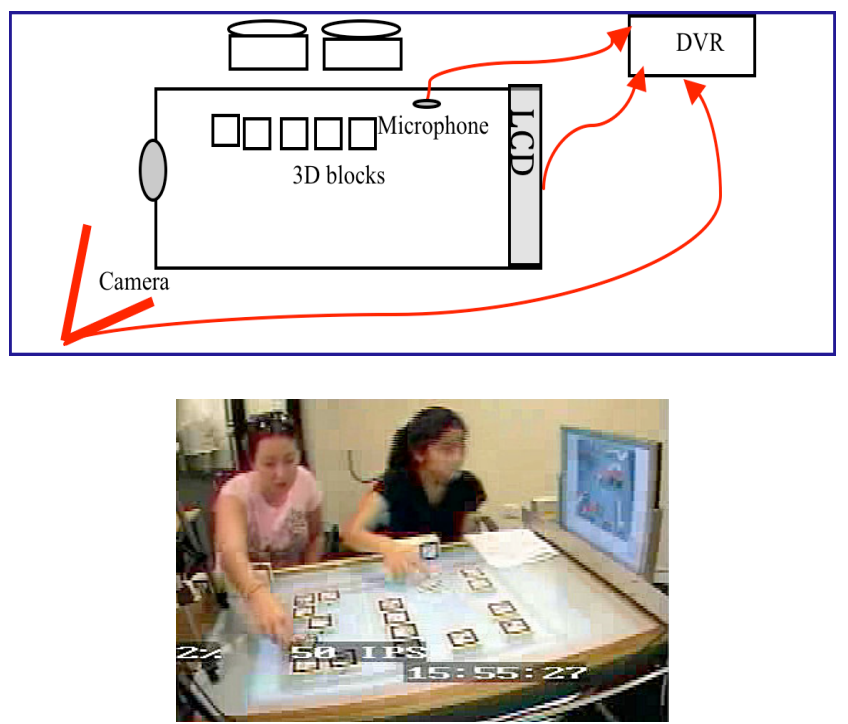

Table 5 shows the set-up for the GUI sessions. The overall experiment set-ups were similar to those of the TUI sessions. However, the camera was set to the left of the designers to avoid the LCD screen set to the front of the table from blocking its view of the designers. A pair of designers sat together in front of the computer, and the designer on the right usually operated the mouse.

Designers in the TUI sessions communicated design ideas by moving the objects visually, whereas designers in the GUI sessions discussed ideas verbally. Further, designers in the TUI sessions collaborated on handling the 3D blocks more interactively whereas designers in the GUI sessions shared a single mouse, thus one designer mainly manipulated the mouse while the other explained what $\mathrm{s} / \mathrm{he}$ was focusing on. These findings suggest that designers' collective interactions differed in the two design sessions. 
TABLE 5. Experiment setting for GUI session (Kim and Maher, 2008)

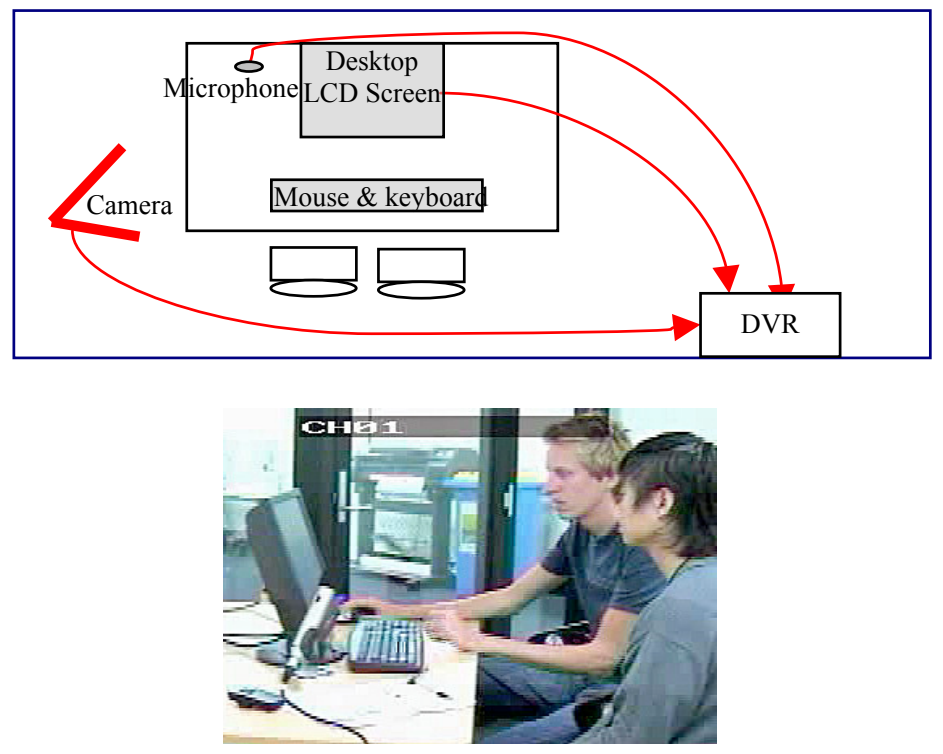

It is notable that designers of the TUI sessions often kept touching the 3D blocks, and designers of the GUI sessions showed similar touching actions using the mouse. 'Touch' actions did not accompany any change in the placement of objects, but seemed to involve a cognitive process. Kim and Maher (2008) conjectured that 'Touch' gestures supported designers' perception of visuo-spatial features based on the argument by Kirsh and Maglio (1994): Some actions that appear unmotivated actually play valuable roles in improving performance, for instance, simplifying mental computation, from a perspective of epistemic goals.

Designers in the TUI sessions randomly placed pieces of furniture on the horizontal display of the plan, and then decided on their locations by moving them around. They were acting spontaneously, responding to their perceptual information straight away. On the other hand, designers in the GUI sessions seemed to decide their actions based on the information initially given rather than perceptual information. For example, regarding the placement of a new desk, designers in the GUI sessions emphasized the function of a desk for a computer programmer by saying "the programmer 
Designers and Collaborative Virtual Environments

might need a desk for holding little computer things" and placing it in the corner. However, designers in a TUI session considered two locations for the desk, in the corner or near the window, then deciding to put it near the window so that the designer could look out, thus creating a spatial relationship between the desk and window. These findings suggest that designers developed design ideas in different ways according to the different interaction modes.

Through the results of the experiments, Kim and Maher (2008) found that the physical interaction with objects in TUIs produce epistemic actions as an 'exploratory' activity to assist in designers' spatial cognition. Further, the epistemic 3D modeling actions afforded by the interface offload designers' cognition, and the naturalness of the direct hands-on style of interaction promote designers' immersion in designing, thus allowing them to perform spatial reasoning more effectively. In addition, designers' perception of visuo-spatial information, especially 'spatial relations', was improved while using the 3D blocks. The simultaneous generation of new conceptual thoughts and perceptual discoveries when attending to the external representation may also be explained by a reduction in the cognitive load of holding alternative design configurations in a mental representation.

In terms of the design process, designers' problem finding behaviours were increased in parallel with the change in designers' spatial cognition. The 'problem-finding' behaviours and the process of re-representation provided the designers with deeper 'insight' leading to key concepts for creative design. In summary, the study provides empirical evidence for the following views on TUIs: firstly, TUIs change designers' spatial cognition, and secondly, the changes of the spatial cognition are associated with problem finding behaviours typically associated with creative design processes.

\section{Adaptive Virtual Worlds}

While most developments and studies of CVEs focus on how to support collaboration, little research attention is given to the design of the virtual world as a place. Since a CVE is a virtual world, we can ascribe behaviours to the objects in the world in addition to designing their geometry and location in the world. This provides an opportunity to rethink how places are designed in CVEs. Gu and Maher (2005) present an 
approach to designing places in CVEs that are responsive to the needs of the people in the world and automatically adapt to changing needs.

Typically, fixed behaviours are ascribed to $3 \mathrm{D}$ objects in a virtual world. Maher and Gero (2002) propose a multi-agent system, shown in Figure 1, to represent a 3D virtual world so that each object in the world has agency. With sensors and effectors as the interface to the 3D virtual world, each agent can sense the world, reason about the goals and modify the virtual world to satisfy the goals. 3D virtual worlds developed using this model can adapt their designs to suit different needs.

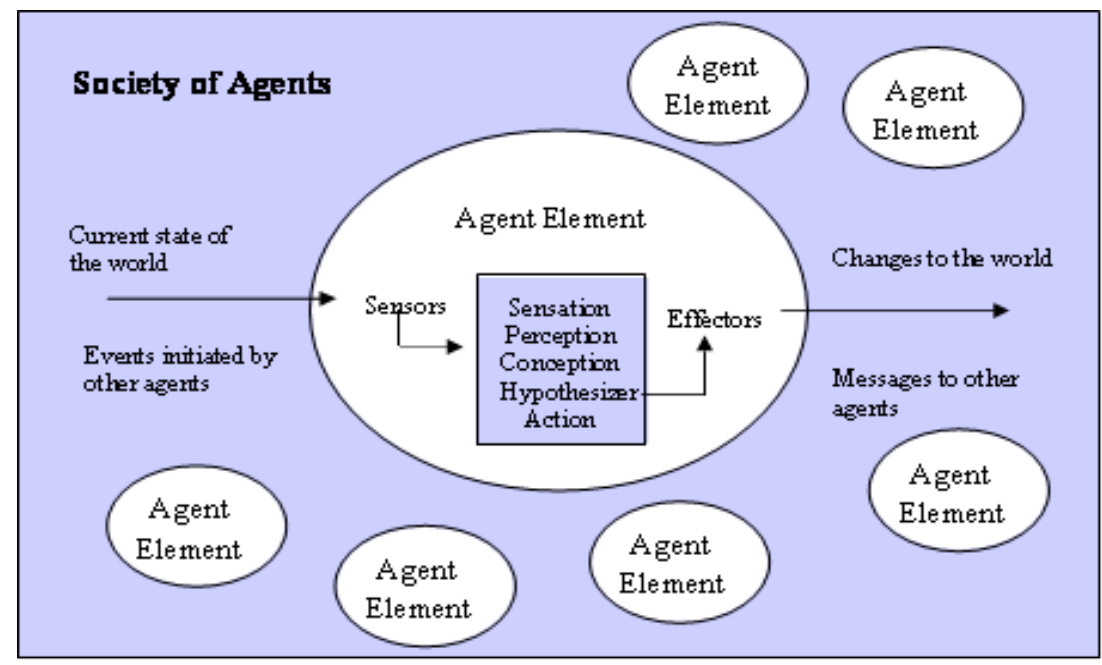

Figure 1. A 3D virtual world as a multi-agent system (Maher and Gero 2002)

Maher and $\mathrm{Gu}$ (2005) developed this agent model further to automatically generate and adapt a 3D virtual world. The generative design agent (GDA) is an agent whose core processor is a design grammar. In addition to a world agent, each object in the world has agency and is capable of modifying itself. The rules in the grammar were grouped to provide the following design functions:

- Layout rules to identify functional spaces.

- Object rules to select and place the objects that enable the functions. 
- Navigation rules to select and specify navigation methods.

- Interaction rules to select and ascribe behaviours to objects.

The GDA model enables 3D virtual worlds to be dynamically designed as needed. As the core of a GDA's design component, the generative design grammar includes the representation of design context of $3 \mathrm{D}$ virtual worlds in the forms of state labels, which can be used to match against the GDA's current interpretation for directing the grammar application. This research provides new insights for 3D virtual worlds from the following perspectives:

The GDA model introduces dynamics and autonomy to the designs of 3D virtual worlds. Virtual worlds designed with the GDA model do not have a static infrastructure like built environments. The CVE is designed for a particular "moment", and reflects its inhabitants' needs of that "moment".

The generative design grammar framework serves as a base for developing generative design grammars with different styles that suits different design purposes. This means that different agents can capture different styles that can be modified, combined, and evolved.

The generative design grammar framework also provides a foundation to formally study the styles of 3D virtual worlds. Compared to other novice designs, virtual worlds designed with a specific style in mind will achieve better consistency in terms of visualisation, navigation and interaction, and this consistency provides a strong base to assist its occupants' orientations and interactions in the virtual worlds.

\section{Summary}

This chapter provides an overview of the ways in which CVEs provide a unique approach to supporting collaborative design. The concept of a CVE differs from more traditional collaborative tools by providing an immersive sense of place in a multi-user 3D virtual world. Following a presentation of the technical and social issues in using CVEs for collaborative design, the chapter provides an overview of three research projects that consider the impact of CVEs on the collaborative design process, the impact of new interaction technologies on the collaborative design process, and the potential for CVEs to provide a new kind of material for designing virtual places. The use of CVEs for collaborative 
design facilitates the seamless movement from working closely on the same task to working individually on a complex shared design task. The use of new interface technologies, such as tangible user interfaces, has the potential to redirect the focus of the designers on spatial relationships rather than the geometry of the parts, and to facilitate problem finding behaviour. Finally, as a new design material, CVEs allow designers to create proactive and adaptive places that respond to the changing needs of the avatars.

\section{References}

Azuma, R, Baillot, Y, Behringer, R, Feiner, S, Julier, S and Maclntyre, B: 2001, Recent advances in augmented reality, Computer Graphics and Applications, 21(6): 34-47.

Azuma, RT: 1997, Survey on augmented reality, Presence: Teleoperators and Virtual Environments, 6(4): 355-385.

Bekker, M, Olson, JS and Olson, GM: 1995, Analysis of gestures in face-to-face design teams provides guidance for how to use groupware in design, The Symposium on Designing Interactive Systems (DIS)'95, pp. 157-166.

Benford, S, Greenhalgh, C, and Rodden, T: 2001, Collaborative virtual environments, Communications of the ACM, 44(7): 79-85.

Billinghurst, M, Kato, H and Poupyrev, I: 2000, ARToolKit: a computer vision based augmented reality toolkit, IEEE VR2000, New Jersey.

Churchill, EF, Snowdon, DN, and Munro, AJ (eds): 2001, Collaborative Virtual Environments: Digital Places and Spaces for Interaction, Springer-Verlag, London.

Clark, S and Maher, M: 2006, Collaborative learning in a 3D virtual place: Investigating the role of place in a virtual learning environment, Advanced Technology for Learning, 3(4): 208-0896.

Daruwala, Y: 2004, 3DT: Tangible input techniques used for 3D design \& visualization, Masters Thesis, The University of Sydney.

Dias, JMS, Santos, P, Bastos, L, Monteiro, L, Silvestre, R and Diniz N: 2002, MIXDesign: Tangible mixed reality for architectural design, 1st Ibero-American Symposium in Computer Graphics (SIACG).

Gabriel, GC: 2000, Computer Mediated Collaborative Design in Architecture: The Effects of Communication Channels on Collaborative Design Communication, $\mathrm{PhD}$ Thesis, Architectural and Design Science, Faculty of Architecture. Sydney, University of Sydney.

Gero, JS and McNeill, T: 1998, An approach to the analysis of design protocols, Design Studies 19(1): 21-61.

$\mathrm{Gu}, \mathrm{N}$ and Maher ML: 2005, Dynamic designs of 3D virtual worlds using generative design agents, in B Martens and A Brown (eds), Computer Aided Architectural Design Futures 2005, Springer, Dordrecht, Netherlands, pp 239-248. 
Designers and Collaborative Virtual Environments

Gul, LF and Maher, ML: 2009, Co-creating external design representations: comparing face-to-face sketching to designing in virtual environments, CoDesign International Journal of CoCreation in Design and the Arts, 5(2): 117-138.

Kim, MJ and Maher, ML: 2008, The impact of tangible user interfaces on spatial cognition during collaborative design, Design Studies, 29(3): 222-253.

Kirsh, D and Maglio, P: 1994, On distinguishing epistemic from pragmatic action, Cognitive Science, 18:513-549.

Kvan, T: 2000, Collaborative design: What is it? Automation in Construction, 9(4): 409415.

Kvan, T and Candy, L: 2000, Designing collaborative environments for strategic knowledge in design, Knowledge-Based Systems, 13: 429-438.

Maher, ML, Rosenman, M, Merrick, K and Macindoe, O: 2006, DesignWorld: An augmented 3D virtual world for multidisciplinary collaborative design, in A Kaga and R Naka (eds), CAADRIA '06: Proceedings of the 11th Conference on Computer-Aided Architectural Design Research in Asia, Osaka, Japan, pp. 133-142.

Maher, ML, Bilda, Z and Marchant, D: 2005, Comparing collaborative design behavior in remote sketching and 3D virtual worlds, Proceedings of International Workshop on Human Behaviour in Designing, Key Centre of Design Computing and Cognition, University of Sydney, pp. 3-26.

Maher, ML and Gero, JS: 2002, Agent models of 3D virtual worlds, ACADIA 2002: Thresholds, California State Polytechnic University, Pomona, pp. 127-138.

McGrath, A \& Prinz, W: 2001, All that is solid melts into software, in EF Churchill, DN Snowdon, and AJ Munro (eds), Collaborative Virtual Environments: Digital Places and Spaces for Interaction, Springer-Verlag, London.

Redfern, S and Naughton, N: 2002, Collaborative virtual environments to support communication and community in internet-based distance education, Journal of Information Technology Education, 1(3): 201-211.

Saad, M and Maher, ML: 1996, Shared understanding in computer-supported collaborative design, CAD Journal, B(3): 183-192.

Scott, SD, Grant, KD and Mandryk, RL: 2003, System guidelines for co-located, collaborative work on a tabletop display, Proceedings of the European Conference Computer-Supported Cooperative Work, pp. 159-178.

Streitz, NA, Geißer, J, Holmer, T, Konomi, S, Muller-Tomfelde, C, Reischl, W, Rexroth, P, Seitz, P and Steinmetz, R: 1999, i-Land: An interactive landscape for creativity and innovation, the Conference on Human Factors in Computing Systems (CHI'99), pp. 120-127.

Tang, JC: 1991, Findings from observational studies of collaborative work, International Journal of Man-Machine Studies, 34: 143-160.

Ullmer, B and Ishii H: 1997, The metaDESK: Models and prototypes for tangible user interfaces, Proceedings of User Interface Software and Technology (UIST)' 97. 


\section{Biography}

Mary Lou Maher is a Program Director in the Information and Intelligent Systems Division at NSF and an Honorary Professor in the Design Lab at the University of Sydney. She joined the Human Centered Computing Cluster at NSF in July 2006 and started the CreativeIT program. Her research includes the development of cognitive and computational models of design and collaboration. She uses the protocol analysis method to demonstrate how new technologies impact design, collaboration, education, and creativity. She has studied the use of virtual worlds for education including MOOs, web-based learning environments, and 3D multi-user virtual worlds. 